Research Article

\title{
Understanding the Ion Exchange Process in LDH Nanomaterials by Fast In Situ XRPD and PCA-Assisted Kinetic Analysis
}

\author{
Luca Palin, ${ }^{1,2}$ Marco Milanesio $\left(\mathbb{D},{ }^{1}\right.$ Wouter van Beek, ${ }^{3}$ and Eleonora Conterosito $\mathbb{D}^{1}$ \\ ${ }^{1}$ DiSIT, Università del Piemonte Orientale, Via Michel 11, I-15121, Italy \\ ${ }^{2}$ Nova Res s.r.l., Via D. Bello 3, I-28100 Novara, Italy \\ ${ }^{3}$ Swiss-Norwegian Beamlines, ESRF, The European Synchrotron, CS40220, Grenoble 38043, France \\ Correspondence should be addressed to Eleonora Conterosito; eleonora.conterosito@uniupo.it
}

Received 4 October 2018; Accepted 30 December 2018; Published 25 February 2019

Academic Editor: Domenico Acierno

Copyright (c) 2019 Luca Palin et al. This is an open access article distributed under the Creative Commons Attribution License, which permits unrestricted use, distribution, and reproduction in any medium, provided the original work is properly cited.

\begin{abstract}
Layered double hydroxides (LDHs) are nanomaterials with interesting properties finding applications in many fields, such as catalysis, environmental chemistry, and pharmaceuticals. They are anionic clays with positively charged layers and anions within the layers to reach neutrality. Their properties are defined by both composition and morphology. The composition can be tuned by exchanging the interlayer anion. The far more stable, common, and highly prevalent among natural LDHs is the carbonate anion thanks to its double negative charge. To adapt the properties of LDHs for technological applications, the challenge is to exchange the carbonate with the functionalizing monovalent anions in an effective and cheap way. In this study, the exchange of carbonate with nitrate ions is studied by in situ X-ray powder diffraction (XRPD). The exchange is carried out by a liquid-assisted grinding approach, inserting the mechanically ground dry sample in a capillary and then wetting it with a drop of nitric acid, while measuring the XRPD pattern. The kinetics of the process was investigated by the Avrami-Erofe'ev method; the reaction mechanism was determined using the advancing interface model and by analyzing the XRD peak shapes, which evidentiate changes in the crystallinity during the reaction. The reaction starts from the faces perpendicular to the layers and occurs along the channels, increasingly limited by diffusion when approaching the internal part of the crystals.
\end{abstract}

\section{Introduction}

Layered double hydroxides (LDHs) have attracted a lot of interest in the last years $[1,2]$ thanks to the possibility of hosting different inorganic or organic anions that can be intercalated between the mixed metal (e.g., $\mathrm{Zn} / \mathrm{Al}$ or $\mathrm{Mg} / \mathrm{Al}$ ) hydroxide layers to counterbalance the positive charge of the layer. They find applications in many fields, from pharmaceutical or cosmetic preparations [3-9] to catalysis [10-13] and polymer additives [14-16] to adsorbents for decontamination [17-20]. The vast possibility of applications in the industry is driving the research toward finding new facile and economically profitable ways to obtain functionalized hydrotalcites [21]. The majority of hybrid hydrotalcites are obtained by ionic exchange [21] starting from a nitrate or chloride hydrotalcite, while the most common and cheap
LDH contains the carbonate anion which is very stable. The reason is that the far larger charge density of carbonate with respect to nitrate, increasing the interaction with the inorganic layer, hinders the exchange reaction. To allow an easy and scalable preparation method for organic hydrotalcites, an abundant source of nitrate hydrotalcite is required. Iyi et al. [22] proposed a method based on nitric acid ethanol-containing solution. This method was then adapted by performing it in a quasi-solid state, similar to the liquid-assisted grinding (LAG) method developed by some of us $[15,23,24]$. The mechanism of this reaction and its kinetics must be deeply investigated to further understand the process and improve its yield, while, at the same time, reducing the amount of solvents, wastes, and byproducts. Synchrotron-based X-ray diffraction studies [25-28] can be very helpful in elucidating solid state reaction mechanisms 
and/or kinetics, especially when supported by approaches able to efficiently and selectively analyze the in situ XRPD data [29]. To fully characterize this almost solid state reaction, a setup proposed by some of us $[30,31]$ was optimized and exploited to study by in situ X-ray powder diffraction (XRPD) the exchange of carbonate LDH to obtain nitrate LDH. Understanding the parameters that govern the exchange mechanism is in fact vital for optimizing the nitrate $\mathrm{LDH}$ production process. A commercial carbonate $\mathrm{LDH}$ sample was chosen to fit the real world case. The exchange of carbonate with nitrate ions into LDH is hence studied by in situ XRPD, allowing kinetic, reaction mechanism, and microstructural information to be extracted. The reaction kinetics were analyzed by the Avrami-Erofe'ev approach to obtain the reaction order by the traditional refinement approach [27, 28, 32, 33] and by the recently proposed PCA-based approach [24, 34-36].

\section{Materials and Methods}

The starting materials are the PURAL ${ }^{\circledR} \mathrm{MG} 63 \mathrm{HT}$, a $\mathrm{Mg} / \mathrm{Al}$ carbonate hydrotalcite $\left(\mathrm{Al}_{2} \mathrm{O}_{3}: \mathrm{MgO}\right.$ 37:63 wt\%. ratio, $\mathrm{CO}_{3}{ }^{2-} 10 \mathrm{wt} \%$.) purchased from SASOL (http://www. sasoltechdata.com). Nitric acid and ethanol were purchased from Sigma (Milano, Italy). All chemicals and solvents were reagent grade and used without further purification.

The synchrotron XRPD data were collected at ESRF on the Swiss-Norwegian Beamline with the standard BM1B setup [37], using a wavelength of $0.70158 \AA$. The BM1B optics provides an $\mathrm{X}$-ray beam spot of 1 (horizontal) $\times 1$ (vertical) $\mathrm{mm}$ at the sample position. The diffractometer was especially equipped with a Pilatus 2M [38] X-ray detector for these experiments. The detector has a pixel size of $172 \times 172 \mu \mathrm{m}$ and was placed at $1430 \mathrm{~mm}$ from the sample. The setup and calibration procedures are described in Ref. [39] by van Beek et al.

The reactions were carried out in a capillary, using the setup described in detail in Ref. [31] by Conterosito et al. and monitored by XRPD with a Debye-Scherrer geometry. Part of the dry grinded $\mathrm{LDH}_{-} \mathrm{CO}_{3}$ sample was put in a capillary between two glass fiber flocks. One end was left open and the other was connected to a vacuum pump. On the free end, far from the sample, a drop of ethanol/ $\mathrm{HNO}_{3}$ solution [22] (weight composition: 7.28\% $\mathrm{HNO}_{3}, 3.92 \% \mathrm{H}_{2} \mathrm{O}$, and $88.79 \% \mathrm{EtOH}$ ) was deposed. After the collection of some patterns at "time zero," the $\mathrm{HNO}_{3}$ solution was brought in contact with the mechanical mixture by pulling it in with a vacuum pump standing outside the experimental hutch. A complete powder pattern was collected every $0.1 \mathrm{~s}$. In order to carry out kinetic analysis, data were recorded continuously until the reaction was estimated to be complete. The experiment was stopped when no or negligible changes in XRPD peak intensities were observed in real time.

In situ XRPD data were analyzed to obtain the reaction coordinate at first by PCA analysis [34-36, 40] for a fast and efficient identification of the patterns during which the reaction occurs. Then the more interesting subset was analyzed by Pawley refinement using TOPAS [41] software to obtain an accurate description of the reaction coordinate. The kinetic analysis was carried out by the Avrami-Erofe'ev and the advancing interface equations [42]. The Avrami-Erofe'ev approach is a generic "reaction model" only related to the dimensionality (1-, 2-, and 3D reactions or $<1 \mathrm{D}$ if diffusion limited) of the reaction mechanism description. Conversely, the advancing interface model implies a geometrical description of the advancing front of the reaction, representative of what happens within the crystallites at the molecular and nanometric level.

Textural and morphological observations (secondary electron (SE) images) were performed by means of a Tescan FE-SEM (Mira 3XMU-series). The operating conditions were as follows: $20 \mathrm{kV}$ accelerating voltage, around $13 \mathrm{~mA}$ beam current, and different working distance and magnifications (reported in each photo). Samples for SEM observations were prepared by C-coating from graphite evaporation.

\section{Results and Discussion}

3.1. Analysis of In Situ XRPD Data. The XRPD data, collected as detailed in the experimental section, were integrated and normalized using the incident intensity given by monitor count of the beamline. A first exploratory analysis using the PCA approach, as implemented in RootProf [43], allowed to check the data quality and promptly individuate the region of interest. As often occurring during in situ experiments, to get the starting part of the reaction, the data collection is started before the operations needed to induce the reaction. Therefore, there is a preperiod for the reaction of unknown length and the data collection is much longer than the reaction time. In fact, to avoid losing the final part of the reaction, the data collection was continued far beyond the real end of the experiment. This is especially true for fast reactions evolving within seconds and ending in about one minute. Moreover, the experiment was repeated some times to optimize the setup and conditions. This results in many datasets of thousands of patterns each. Therefore, a preliminary PCA analysis was performed on the datasets to guide the selection of the best run and of the significant patterns within, as described in Section 3.1.1. The visual inspection of the selected patterns (one out of the 10 in the first hundred and then 1 out of 100) reported in Figure 1(b) highlights two aspects. On the one hand, the expected changes of intensity of the reactant and product main peaks are observed with the FWHM remaining rather small, thus indicating that the reaction proceeds mainly at the solid state. On the other hand, a relevant change in the background, with a bump centered at $2 \theta \approx 10^{\circ}$ below the 006 reflection of hydrotalcite, suggests the presence of a low-ordered fraction, probably amorphous mixed oxides originated by $\mathrm{LDH}$ dissolution mixed with the solution within the capillary.

3.1.1. PCA Analysis on Raw XRPD Data. PCA analysis, as demonstrated recently by some of us [24, 34-36], can unravel with a blind (without any knowledge of the crystal structure or other a priori information) and efficient approach, the evolution and the kinetics occurring during an in situ experiment. Summarizing, PCA scores are related to the kinetics of 


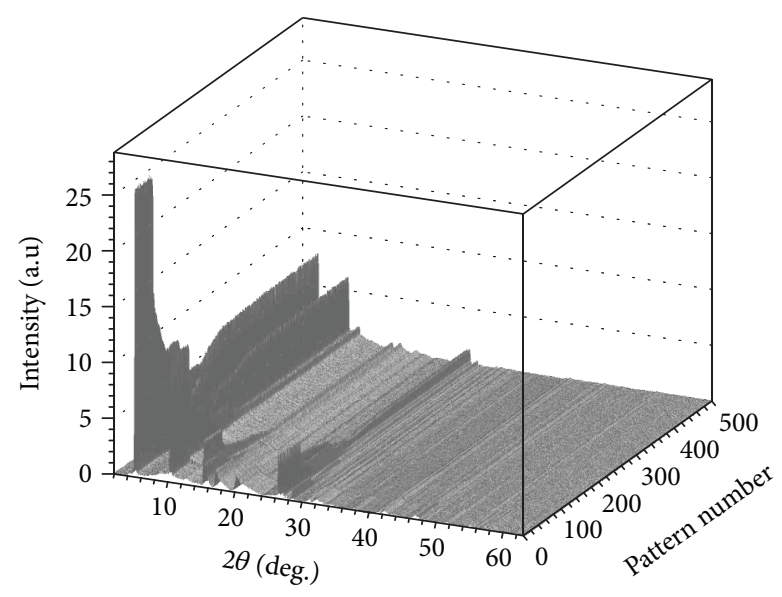

(a)

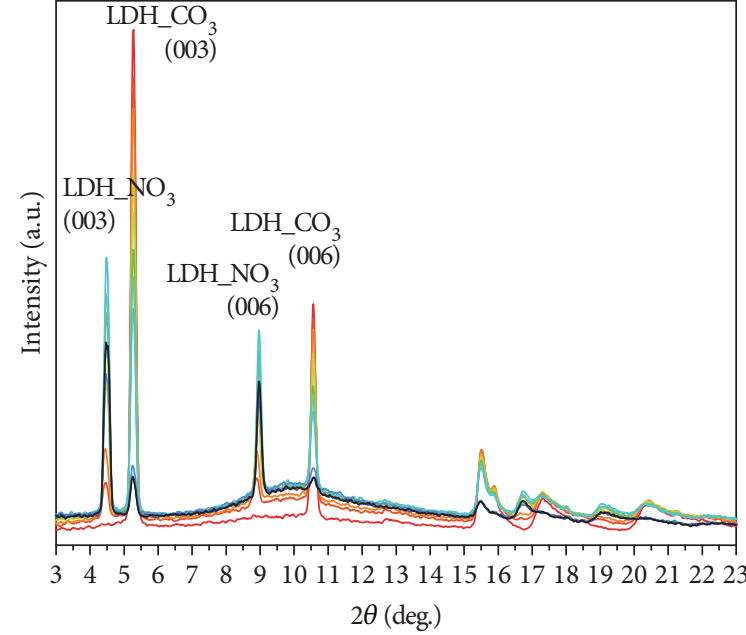

$\begin{array}{rrrr}-48 & -60 & -90 & -300 \\ -49 & -70 & -100 & -400 \\ -50 & -80 & -200 & \end{array}$

(b)

Figure 1: 3D plot of the first 500 of the 2399 XRPD collected patterns (a) and plot of selected XRPD patterns (b).
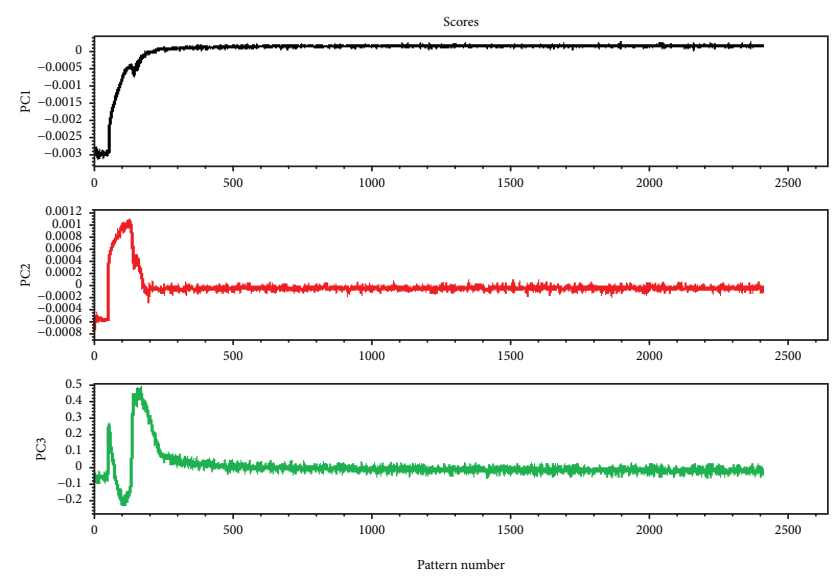

(a)
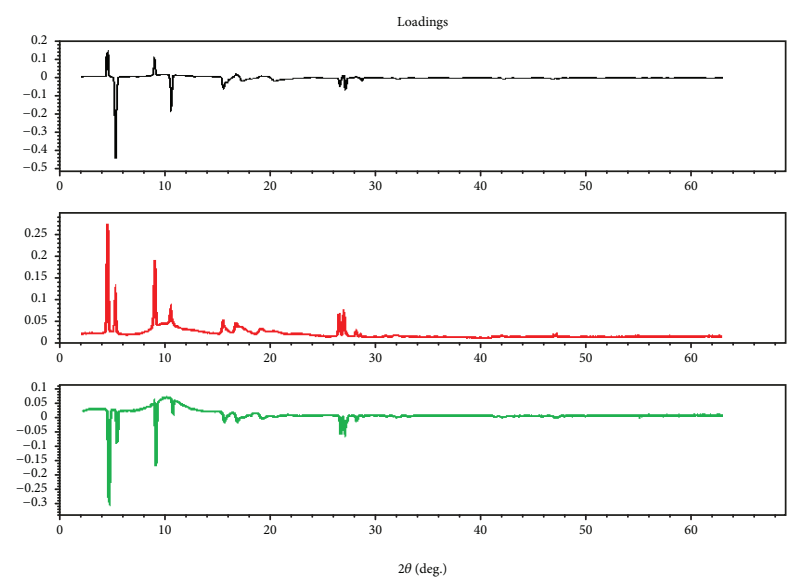

(b)

Figure 2: Plot of PCA scores (a) and loadings (b) of the first three PCs from the analysis of the full dataset. The explained variance is $\mathrm{PC} 1=83.19 \%, \mathrm{PC} 2=12.84 \%$ (overall 96.0\%), and PC3 $=1.83 \%$ (overall 97.9\%).

the reaction, while PCA loadings give indication of what components of the original XRPD pattern are described by each PC. As an example, PCn scores give the kinetics related to the XRPD peaks visible in PCn loadings. The number of PCs depends on the richness of variance of the system. The number of PCs to be considered depends on the \% of explained variance (given in the captions of Figures 2 and 3). Typically, for in situ XRPD, 2 to 3 PCs are enough to describe the accepted threshold of $95 \%$ of the variance.

PCA was therefore exploited without performing any preprocessing of the data (Figure 2) to individuate the XRPD patterns where the reaction occurs, to check the data quality, and to have a preliminary view of the evolution observed during the reaction. PCA scores vs. time plot represents the variance over time in the dataset. Since the variance is due to the variations of the XRPD patterns because of the carbonate to nitrate exchange, it gives the trend of the reaction. After a preperiod, the reaction started at pattern \#48, accelerated until about pattern \#65, and was at equilibrium by pattern \#265. The loadings instead are the key to understand the meaning of the trend represented by the scores. The plot of the loadings resembles the powder pattern so the phases can be recognized. The peaks following the trend of the scores are positive while the ones that are anticorrelated to that trend are negative. In view of these considerations, the preliminary blind PCA analysis suggested a good data quality, without any 

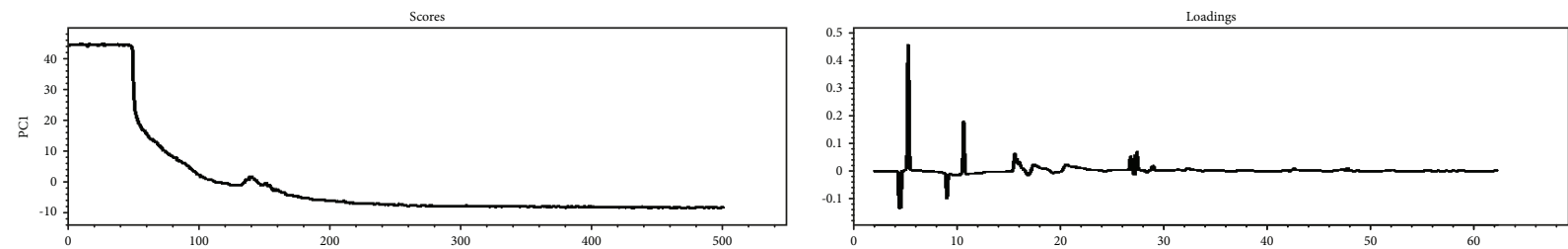

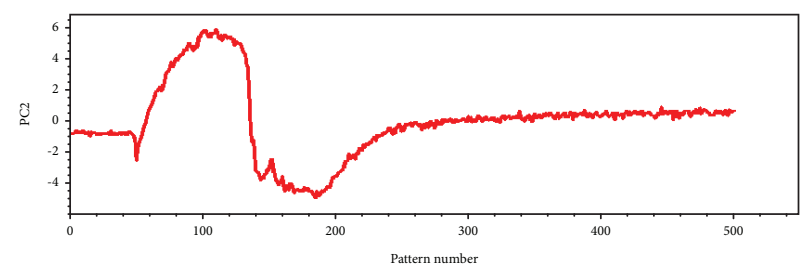

(a)

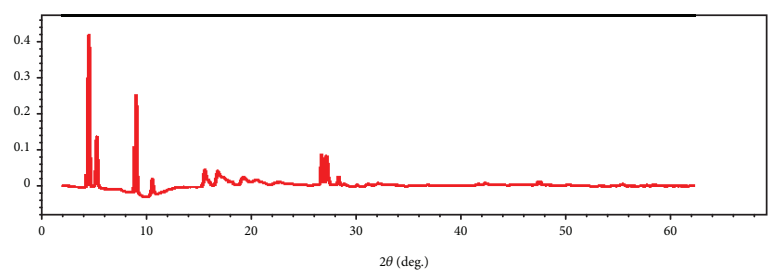

(b)

FIGURE 3: Plot of PCA scores (a) and loadings (b) of the first two PCs with preprocessing applied. The explained variance is PC1 $=97.04 \%$ and PC2 $=2.12 \%$ (overall 99.2\%).

spurious species except carbonate and nitrate LDH. PCA analysis was then repeated reducing the range (from pattern \#0 to \#500) and applying the standard preprocessing for XRPD data.

The patterns were normalized, and background removal was performed using the SNIP algorithm with a window of 100 points. The preprocessing reduced some noise in the data; therefore, two PCs, instead of three, were sufficient to achieve a better explanation of the variance (99\% vs. $98 \%$ ). In this second run (Figure 3), with the number of patterns reduced as suggested by the exploratory PCA (Figure 2), the trend of PC1 is inverted (sign is arbitrary in PCA); therefore, both the score trends and the loadings are inverted (cf. Figures 2 and 3). By looking at the loadings (Figure 3(b)), they are clearly related to the LDH phases showing the main peaks of their XRPD pattern (as can be seen by comparison with Figure 1). The layered peaks of $\mathrm{LDH}_{-} \mathrm{CO}_{3}$ (marked with an asterisk in Figure 3) are positive while those of the $\mathrm{LDH}_{-}$ $\mathrm{NO}_{3}$ phase are negative; therefore, the trend of the scores is correlated to the disappearance of the LDH_CO3 phase and anticorrelated to the appearance of the $\mathrm{LDH} \_\mathrm{NO}_{3}$ phase. These indications suggest that $\mathrm{PC}$, being related to the $\mathrm{LDH} \mathrm{CO}_{3}$ amount, is a good representation of the extent of reaction $\alpha$.

Moreover, in the first 150 patterns, the PCA scores show a complex behavior around pattern 140. These changes are due to abrupt intensity changes in the XRPD patterns, also visible in the raw data (Figure 1). This anomaly is probably due to the $\mathrm{CO}_{2}$ bubbles, produced by the exchange reaction, trying to escape the capillary and creating "voids" and/or liquid-rich regions, varying the amount of sample in the part of the capillary exposed to the X-ray beam.

3.1.2. Peak Fitting Analysis of Raw XRPD Data. In situ XRPD data were then analyzed with a traditional, well-established method of analysis of peak fitting [32, 33] (referred to as peakfit from now on) to check and compare the results of the PCA-based approach. A phase quantification by Pawley fit was performed on the patterns, and the area of the basal peaks of the two phases (reported in Figure 4) was used to

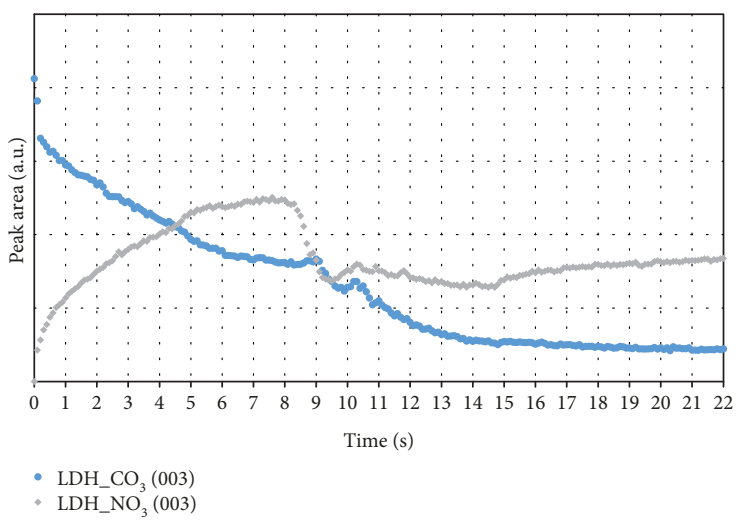

FIGURE 4: Plot of peak area vs. time of the (003) reflection of $\mathrm{LDH}_{-}$ $\mathrm{CO}_{3}$ and $\mathrm{LDH} \_\mathrm{NO}_{3}$ obtained from Pawley fit.

calculate the reaction extent for the decay of the host phase $\left(\alpha_{\text {host }}\right)$ as a function of time $(t)$ by

$$
\alpha_{\text {host }}=\left[\frac{1-\left(I_{\mathrm{h}(t)} /\left(I_{\mathrm{g}(t)}+I_{\mathrm{h}(t)}\right)\right)}{1-\left(I_{\mathrm{h}(\infty)} /\left(I_{\mathrm{g}(\infty)}+I_{\mathrm{h}(\infty)}\right)\right)}\right] \text {, }
$$

where $I_{\mathrm{h}}$ is the area of the (003) peak of the host phase $\left(\mathrm{LDH}_{-} \mathrm{CO}_{3}\right)$ and $I_{\mathrm{g}}$ is the area of the (003) peak of the guest phase (LDH_NO $\mathrm{NO}_{3}$ ) at time $(t)$ and at equilibrium $(\infty)$.

The calculated extent of reaction $\alpha$ is plotted in Figure 5 and compared with the one obtained by PCA. The typical trend of solid state reactions is observed with an acceleration in the very first seconds of the reaction up to the maximum and then the deceleration up to the end of the reaction occurring in about 20 seconds. The shape resembles that of an exponential suggesting a typical diffusion limited model. A negative bump is observed between 8 and 12 seconds, when the reaction already started to decelerate. This bump is visible also in the PCA plots (between patterns \#100 and \#200 (see Figure 2 and its comment)) and is due to $\mathrm{CO}_{2}$ bubble 


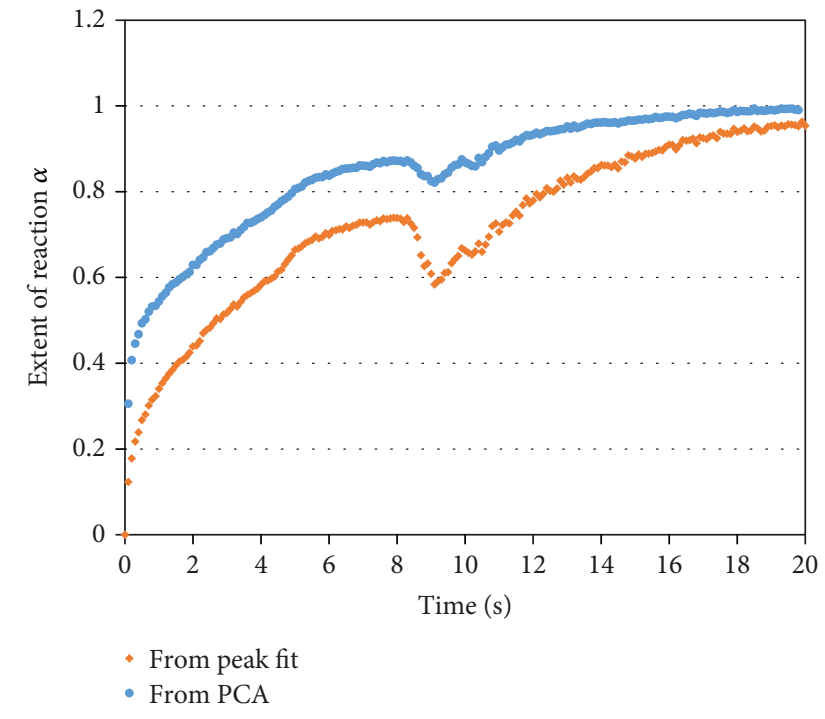

FIGURE 5: Plot of extent of reaction versus time calculated from peakfit (orange diamonds) and from PCA scores (blue circles) of the in situ XRPD data of Figure 1.

formation and/or liquid/solid movements into the capillary. At the end of the reaction, the $\mathrm{LDH}_{-} \mathrm{CO}_{3}$ peaks have not completely disappeared, indicating that the exchange was not complete. This could be due to problems in liquid diffusion and contact with the $\mathrm{LDH}_{-} \mathrm{CO}_{3}$ powder within the capillary, due to its well-known low wettability.

3.1.3. Calculation of the Extent of the Reaction. The PCA scores of Figure 3, representing the kinetic trends of the reaction, were thus used to calculate the reaction extent and compared to that calculated by peak fitting of the (003) peaks of carbonate and nitrate by TOPAS-Academic software. This peak was chosen because it is separated from the band corresponding to the amorphous component, which is superimposed to the 006 peaks (Figure 1(b)) and hence is not a good choice. PCA scores were normalized and inverted according to the fact that the reaction extent should be comprised between 0 and 1 and that we are considering the decrease of the host phase. The two trends (Figure 5) are similar overall but somehow scaled. The difference decreases over time, suggesting that PCA analysis, using the entire XRPD pattern, might give complementary information to the classical XRPD fitting approach. In fact, the difference is more pronounced at the beginning of the reaction when the crystallinity is smaller and amorphous content larger. At the end of the reaction, the PCA- and peakfit-based extent of reaction results (Figure 5) converges to 1 , as expected for the normalization of eq. (1). It must be noted that the peakfit method relies on the a priori information given by the knowledge of the lattice edge of nitrate and carbonate hydrotalcites. This knowledge allows us to concentrate the information extraction from the more sure and reliable part of the pattern. The drawback (besides the increased requirements in terms of human and computer time to integrate and manually fit in the required peaks in all the PCA-selected patterns) is the extraction of only a part of the information given by the
XRPD pattern. The PCA-based method (faster and without any required a priori information) uses the full angular range of the pattern, background, and thus amorphous content, with the drawback of having a limited selectivity in distinguishing the chemical species. Given these considerations, it is hard to rely fully on the PCA or the peakfit approach, giving instead complementary information. The peakfit method is surely related to the crystalline part of the sample, while the PCA-based score can take into account also the amorphous part of the sample.

3.2. Kinetic Analysis by PCA and Traditional Approaches. The reaction extent obtained by PCA and fitting approach (Section 3.1.2 and 3.1.3, respectively) was used to calculate the kinetic parameters by the Avrami-Erofe'ev equation [42]:

$$
\alpha=1-e^{-(k t)^{n}},
$$

and the interpolation of data was performed using its linear form:

$$
\ln [-\ln (1-\alpha)]=n \ln t+n \ln k,
$$

where $\alpha$ is the reaction coordinate (or extent of reaction), $k$ is the rate constant, $t$ is the considered reaction time, and $n$ is the empirical reaction order. A phenomenological model was also used to gain insight into the actual reaction mechanism such as the contracting area model also known as $2 \mathrm{D}$ advancing interface described by [42]

$$
k t=1-(1-\alpha)^{0.5}
$$

that can be expressed in the linear form:

$$
\ln \left[1-(1-\alpha)^{0.5}\right]=\ln k+\ln t .
$$

The Avrami-Erofe'ev plots are reported in Figure 6 and show good agreement values $\left(R^{2}>95 \%\right)$ and similar kinetic parameters, but the advancing interface model gives better linearity. The reaction order is 0.5 . These numbers suggest a $1 \mathrm{D}$ reaction but limited by diffusion. Even if the peak fitting traditional approach is widely accepted, the PCA-based approach uses all the information of the pattern and it cannot be demonstrated that the fitting method must be the benchmark. Concerning the intercept, it is small in both cases (about -1) indicating that nucleation is not a limiting step. In fact, the antilogarithm of the intercept is an estimation of the rate constant, related also to the number of nuclei per volume. The number being very small, the reaction proceeds with a high speed and no limitations due to nucleation.

The kinetic analysis results suggest a 2D interface, advancing in one direction and limited by diffusion. The reaction front is formed by the planes of the faces of the crystallites perpendicular to the layer where the $\mathrm{NO}_{3}{ }^{-}$ions can enter, and the reaction advances then along the channel in the $1 \mathrm{D}$ dimension. At the same time, beyond the front of the $\mathrm{NO}_{3}{ }^{-}$advance, $\mathrm{CO}_{2}$ is evolved with a concurrent and opposite front of $\mathrm{CO}_{2}$ advancing toward the external part of the crystallite. When the reaction front approaches the 


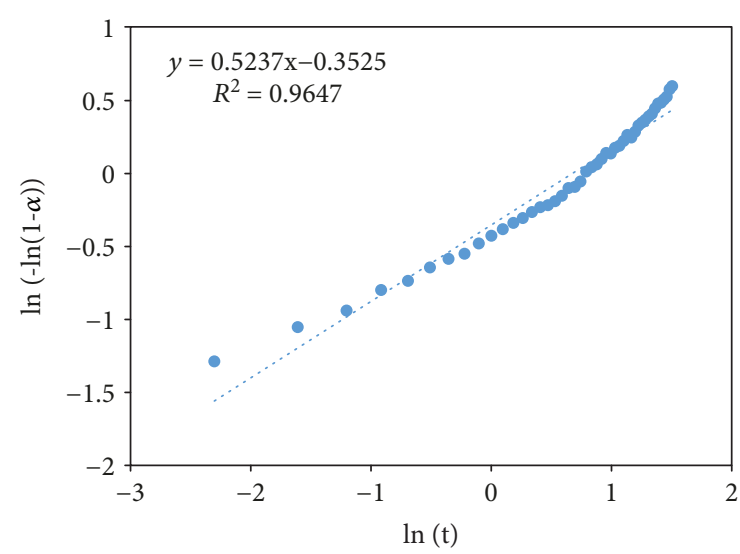

(a)

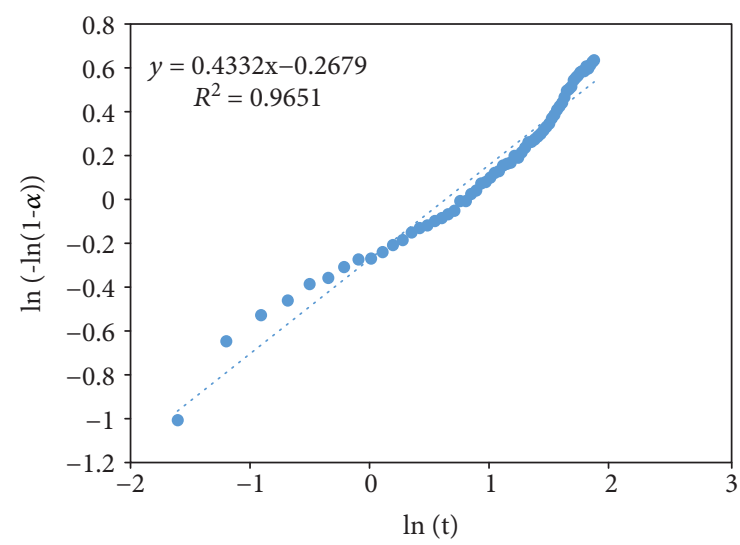

(c)

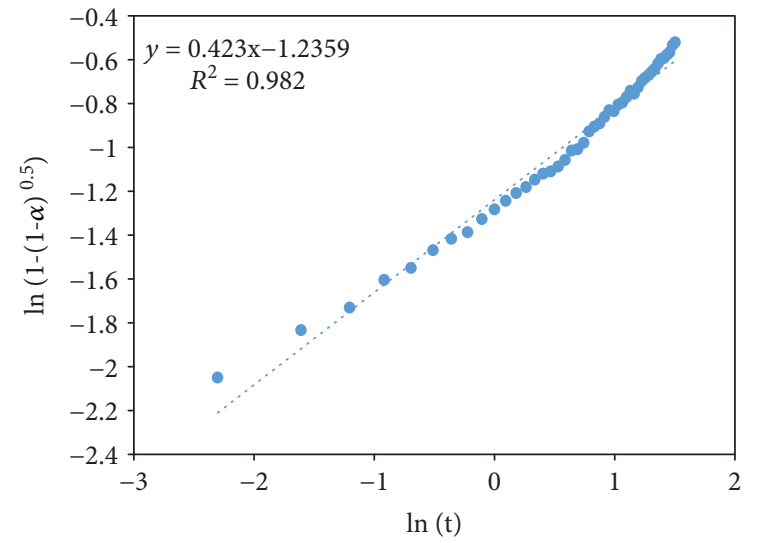

(b)

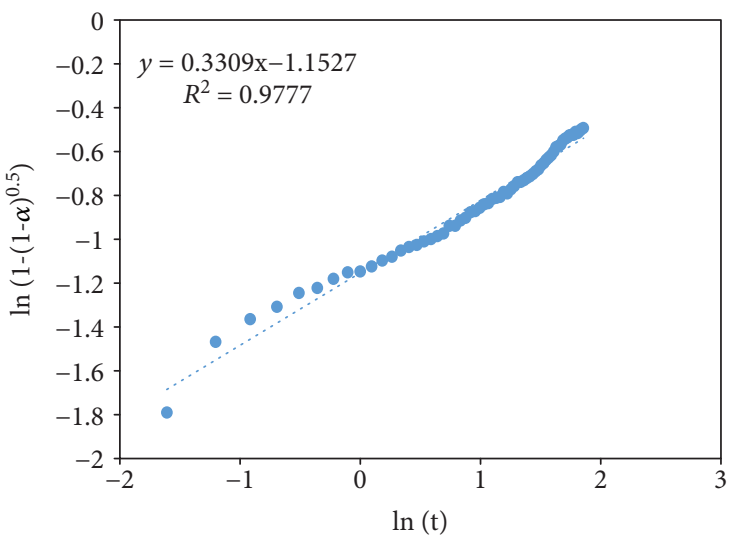

(d)

FIGURE 6: Kinetic analysis: Avrami-Erofe'ev plots with fixed slope for XRPD $(n=1)$ data (a) and PCA@XRPD data (c); 2D advancing interface plots for XRPD data (b) and PCA@XRPD data (d).

center of the crystal, $\mathrm{NO}_{3}{ }^{-}$penetration and $\mathrm{CO}_{2}$ evolution are hampered by diffusion, and thus, the speed is reduced. Looking to Figure 4 (peakfit) and Figure 3(a) (PCA), the two trends are not symmetrical and $\mathrm{LDH}_{-} \mathrm{CO}_{3}$ decrease seems more linear and slower than $\mathrm{LDH} \_\mathrm{NO}_{3}$ formation. This behavior is surely related to the different diffusion coefficients of $\mathrm{NO}_{3}{ }^{-}$and $\mathrm{CO}_{2}$ species within the crystallites. This front, being formed by a mixture of carbonate and nitrate, is less ordered and can be the explanation of the large amorphous band suggested by Figure 1(b). The evolution of this band is also evidenced by PC3 in Figure 2. In fact, PC3 loadings (Figure 2(b)) show this band, and the PC3 scores (Figure 2(a)) show an evident evolution, related to both water and low crystallinity region within the reaction front. The loss of crystallinity is finally confirmed by the crystal size parameter refined by TOPAS TA during peak fitting, reported in Figure 7. The crystallinity of the $\mathrm{LDH} \_\mathrm{NO}_{3}$ phase increases for $8 \mathrm{~s}$ then there is an apparent decrease due to sample movements inside the capillary, with formation of $\mathrm{CO}_{2}$ bubbles as discussed above. The crystallinity of the $\mathrm{LDH} \_\mathrm{NO}_{3}$ phase reaches a plateau after about 35 seconds, after the reaction reaches the equilibrium. The crystallinity of the $\mathrm{LDH}_{-} \mathrm{CO}_{3}$ phase decreases instead.

Finally, it must be noted that a single treatment in the capillary with the hydroalcoholic nitric acid solution is not

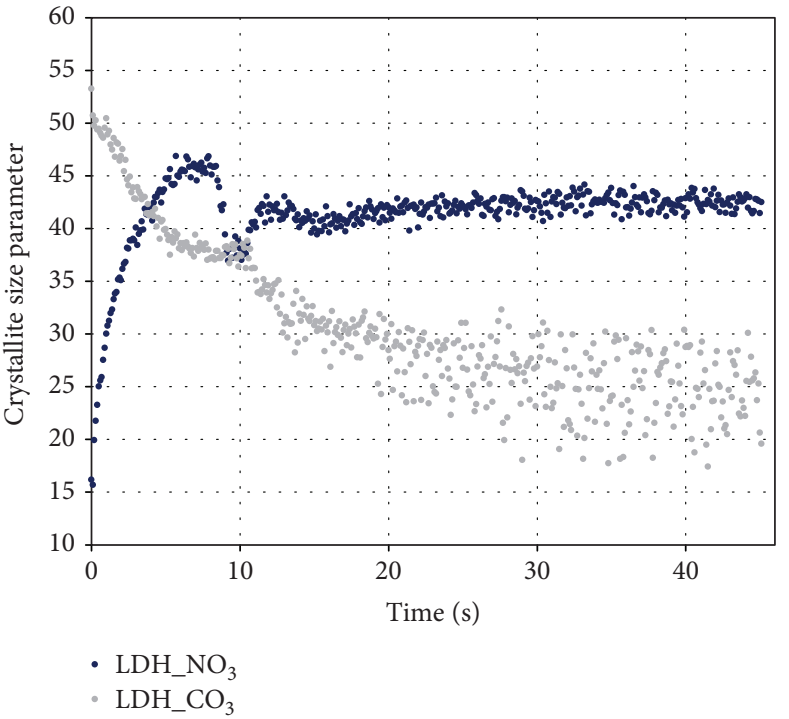

FIGURE 7: Evolution within the reaction of the crystallite size parameter of $\mathrm{LDH}_{-} \mathrm{NO}_{3}$ (dark blue) and $\mathrm{LDH}_{-} \mathrm{CO}_{3}$ (grey) from peak fitting. 


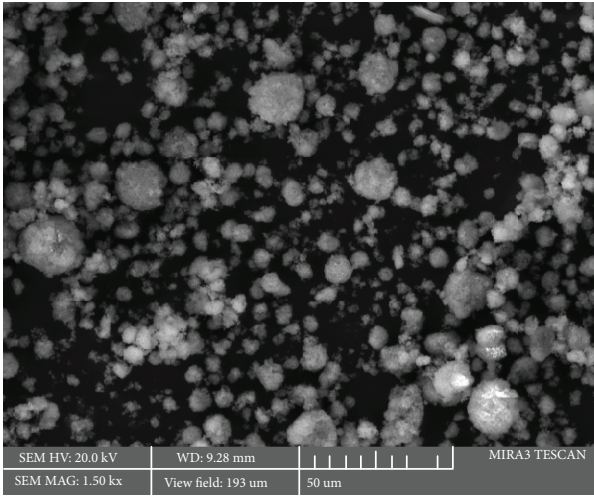

(a)

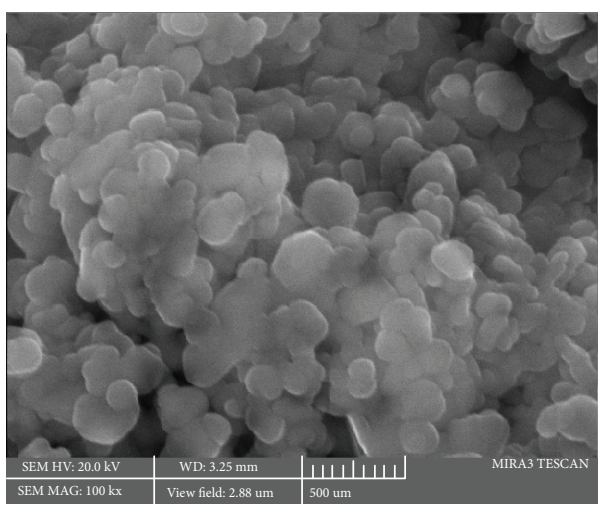

(c)

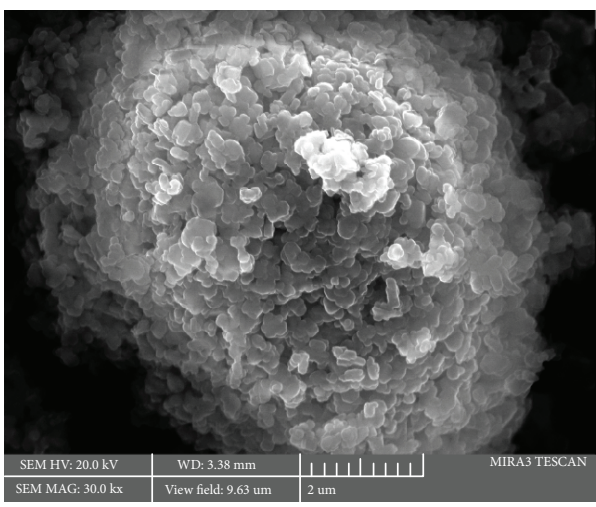

(e)

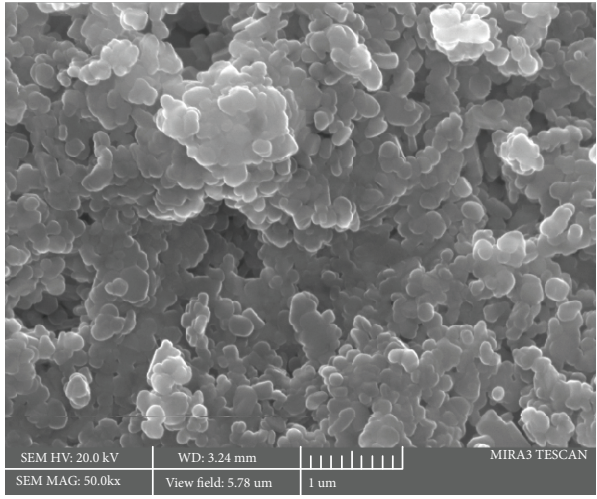

(b)

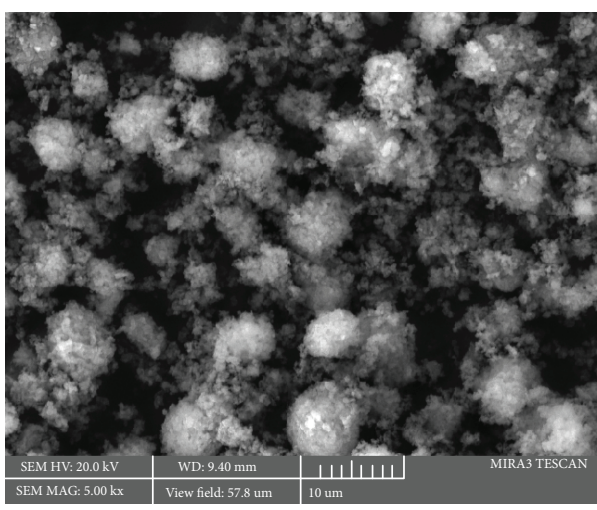

(d)

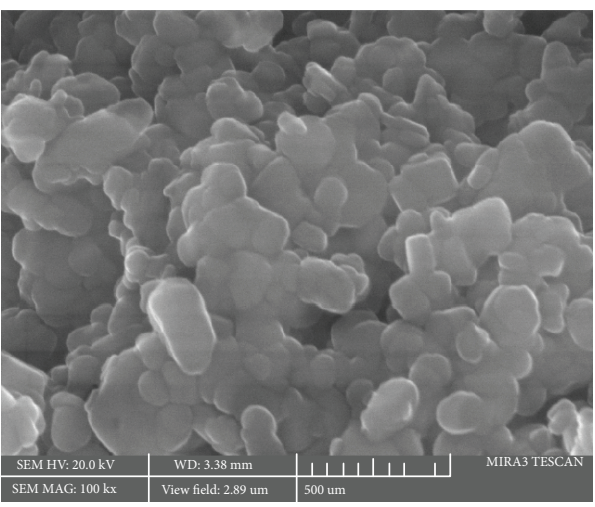

(f)

FIGURE 8: SEM images of $\mathrm{LDH}_{-} \mathrm{CO}_{3}(\mathrm{a}, \mathrm{b}, \mathrm{c})$ and exchanged $\mathrm{LDH}_{-} \mathrm{NO}_{3}(\mathrm{~d}, \mathrm{e}, \mathrm{f})$ at different magnifications.

enough to reach a $100 \%$ yield on conversion to $\mathrm{NO}_{3}-\mathrm{LDH}$. This is probably due to the lack of any blending during the reaction, unlike in the traditional laboratory ex situ approach $[23,44]$. Moreover, a partial dissolution of the $\mathrm{LDH}$ is observed, resulting in an amorphous phase of mixed oxides.

\subsection{Morphologic Analysis by Scanning Electron Microscopy} (SEM). The morphology before and after the carbonate/nitrate exchange into LDH layers was investigated by SEM (Figure 8). The morphology of the two samples is similar, with aggregates of small irregular platelets (Figures $8(\mathrm{a})$, $8(\mathrm{~b}), 8(\mathrm{~d})$, and $8(\mathrm{e}))$. At larger magnifications, the edges of nitrate LDH platelets (Figure 8(f)) seem less sharp and defined than those of carbonate LDH (Figure 8(c)), indicating that probably the amorphous residue shown by XRPD comes from the erosion of the edges by nitric acid. In general, the exchange happens without remarkable changes in the morphology.

\section{Conclusions}

The LDH_CO ${ }_{3}$ to $\mathrm{LDH}_{-} \mathrm{NO}_{3}$ conversion was studied by in situ XRPD. The kinetic analysis indicated that the reaction occurs mainly driven by diffusion with an advancing interface of $\mathrm{NO}_{3}{ }^{-}$entering the crystallites with a speed reduction due to diffusion limitation of the reactant and products. It can be inferred that the reaction proceeds mainly at the solid state with local dissolution phenomena, as suggested by the 
continuous presence of sharp peaks and a moderate bump in the background of the XRPD data collected during the reaction. The reaction is very fast and lasts less than 20 seconds. Efficient $\mathrm{CO}_{2}$ gas evacuation is a key issue for a high yield, continuous, and safe reaction. This is true with a commercial sample with small crystallites and could be even more important in both samples with larger and more regular crystallites or smaller but aggregated crystallites, where diffusion limitation can be even more important. This could be even more important at the real world scale, where the manipulation of LDH can be complicated, because of its sticky nature. This behavior further confirms the need, at the real scale of the lab (grams of product) or of the technological application ( $\mathrm{kg}$ to ton), of a careful sample preparation (drying and gentle milling) and of an efficient mixing of the reactants. From a methodological viewpoint, it must be noted that PCA was confirmed as an efficient method to extract the kinetic information from an in situ XRPD experiment without any a priori knowledge of the structure or even of the sample. Moreover, the information extracted by PCA, using the whole XRPD pattern, is complementary to peakfit and/or Rietveld refinement.

\section{Data Availability}

The in situ XRPD data used to support the findings of this study may be released upon application to Dr. Eleonora Conterosito, who can be contacted at DiSIT, Università del Piemonte Orientale, Via Michel 11, I-15121, Italy (mail to: eleonora.conterosito@uniupo.it).

\section{Conflicts of Interest}

The authors declare no conflict of interest.

\section{Acknowledgments}

Professor Maria Pia Riccardi (Department of Earth and Environmental Sciences, Arvedi Laboratory, CISRIC-University of Pavia) is acknowledged for the SEM analysis. This research is original and had the financial support of the Università del Piemonte Orientale within the RICLOCK project.

\section{References}

[1] C. Del Hoyo, "Layered double hydroxides and human health: an overview," Applied Clay Science, vol. 36, no. 1-3, pp. 103121, 2007.

[2] X. Duan and D. G. Evans, Layered Double Hydroxides, Springer-Verlag, Berlin/Heidelberg, 2006.

[3] L. Perioli, V. Ambrogi, C. Rossi, L. Latterini, M. Nocchetti, and U. Costantino, "Use of anionic clays for photoprotection and sunscreen photostability: hydrotalcites and phenylbenzimidazole sulfonic acid," Journal of Physics and Chemistry of Solids, vol. 67, no. 5-6, pp. 1079-1083, 2006.

[4] V. Ambrogi, G. Fardella, G. Grandolini, M. Nocchetti, and L. Perioli, "Effect of hydrotalcite-like compounds on the aqueous solubility of some poorly water-soluble drugs," Journal of Pharmaceutical Sciences, vol. 92, no. 7, pp. 1407-1418, 2003.
[5] L. Perioli, V. Ambrogi, M. Nocchetti, and C. Rossi, "Effects of hydrotalcite-like nanostructured compounds on biopharmaceutical properties and release of BCS class II drugs: the case of flurbiprofen," Applied Clay Science, vol. 51, no. 4, pp. 407413, 2011.

[6] L. Perioli, V. Ambrogi, B. Bertini et al., "Anionic clays for sunscreen agent safe use: photoprotection, photostability and prevention of their skin penetration," European Journal of Pharmaceutics and Biopharmaceutics, vol. 62, no. 2, pp. 185-193, 2006.

[7] U. Costantino, V. Ambrogi, M. Nocchetti, and L. Perioli, "Hydrotalcite-like compounds: versatile layered hosts of molecular anions with biological activity," Microporous and Mesoporous Materials, vol. 107, no. 1-2, pp. 149-160, 2008.

[8] E. Conterosito, G. Croce, L. Palin et al., "Structural characterization and thermal and chemical stability of bioactive molecule/hydrotalcite (LDH) nanocomposites," Physical Chemistry Chemical Physics, vol. 15, no. 32, pp. 13418-13433, 2013.

[9] M. A. Rocha, P. A. D. Petersen, E. Teixeira-Neto et al., "Layered double hydroxide and sulindac coiled and scrolled nanoassemblies for storage and drug release," RSC Advances, vol. 6, no. 20, pp. 16419-16436, 2016.

[10] D. G. Cantrell, L. J. Gillie, A. F. Lee, and K. Wilson, "Structure-reactivity correlations in $\mathrm{MgAl}$ hydrotalcite catalysts for biodiesel synthesis," Applied Catalysis A: General, vol. 287, no. 2, pp. 183-190, 2005.

[11] U. Costantino, F. Marmottini, M. Sisani et al., "Cu-Zn-Al hydrotalcites as precursors of catalysts for the production of hydrogen from methanol," Solid State Ionics, vol. 176, pp. 2917-2922, 2005.

[12] M. R. Othman, Z. Helwani, and W. J. N. Fernando, "Synthetic hydrotalcites from different routes and their application as catalysts and gas adsorbents: a review," Applied Organometallic Chemistry, vol. 23, no. 9, pp. 335-346, 2009.

[13] S. Ordóñez, E. Díaz, M. León, and L. Faba, "Hydrotalcite-derived mixed oxides as catalysts for different $\mathrm{C}-\mathrm{C}$ bond formation reactions from bioorganic materials," Catalysis Today, vol. 167, no. 1, pp. 71-76, 2011.

[14] C. Taviot-Guého and F. Leroux, "In situ polymerization and intercalation of polymers in layered double hydroxides," Structure and Bonding, vol. 119, pp. 121-159, 2006.

[15] E. Conterosito, I. Benesperi, V. Toson et al., "High-throughput preparation of new photoactive nanocomposites," ChemSusChem, vol. 9, no. 11, pp. 1279-1289, 2016.

[16] Y. Gao, J. Wu, Q. Wang, C. A. Wilkie, and D. O'Hare, "Flame retardant polymer/layered double hydroxide nanocomposites," Journal of Materials Chemistry A, vol. 2, no. 29, article 10996, 2014.

[17] D. Mohan and C. U. Pittman, "Arsenic removal from water/wastewater using adsorbents-a critical review," Journal of Hazardous Materials, vol. 142, no. 1-2, pp. 1-53, 2007.

[18] S. J. Palmer, R. L. Frost, and T. Nguyen, "Hydrotalcites and their role in coordination of anions in Bayer liquors: anion binding in layered double hydroxides," Coordination Chemistry Reviews, vol. 253, no. 1-2, pp. 250-267, 2009.

[19] K. Kuzawa, Y.-J. Jung, Y. Kiso, T. Yamada, M. Nagai, and T. G. Lee, "Phosphate removal and recovery with a synthetic hydrotalcite as an adsorbent," Chemosphere, vol. 62, no. 1, pp. 45-52, 2006.

[20] P. C. Pavan, E. L. Crepaldi, and J. B. Valim, "Sorption of anionic surfactants on layered double hydroxides," Journal of 
Colloid and Interface Science, vol. 229, no. 2, pp. 346-352, 2000.

[21] E. Conterosito, V. Gianotti, L. Palin, E. Boccaleri, D. Viterbo, and M. Milanesio, "Facile preparation methods of hydrotalcite layered materials and their structural characterization by combined techniques," Inorganica Chimica Acta, vol. 470, pp. 36-50, 2018.

[22] N. Iyi, H. Yamada, and T. Sasaki, "Deintercalation of carbonate ions from carbonate-type layered double hydroxides (LDHs) using acid-alcohol mixed solutions," Applied Clay Science, vol. 54, no. 2, pp. 132-137, 2011.

[23] V. Toson, E. Conterosito, L. Palin, E. Boccaleri, M. Milanesio, and V. Gianotti, "Facile intercalation of organic molecules into hydrotalcites by liquid-assisted grinding: yield optimization by a chemometric approach," Crystal Growth \& Design, vol. 15, no. 11, pp. 5368-5374, 2015.

[24] E. Conterosito, M. Milanesio, L. Palin, and V. Gianotti, "Rationalization of liquid assisted grinding intercalation yields of organic molecules into layered double hydroxides by multivariate analysis," RSC Advances, vol. 6, no. 110, pp. 108431108439, 2016.

[25] M. Milanesio, C. Lamberti, R. Aiello, F. Testa, M. Piana, and D. Viterbo, "Iron location in Fe-silicalites by synchrotron radiation single crystal X-ray diffraction," The Journal of Physical Chemistry B, vol. 104, no. 43, pp. 9951-9953, 2000.

[26] L. Palin, C. Lamberti, Å. Kvick et al., "Single-crystal synchrotron radiation $\mathrm{X}$-ray diffraction study of $\mathrm{B}$ and $\mathrm{Ga}$ silicalites compared to a purely siliceous MFI: a discussion of the heteroatom distribution," The Journal of Physical Chemistry B, vol. 107, no. 17, pp. 4034-4042, 2003.

[27] M. Milanesio, G. Artioli, A. F. Gualtieri, L. Palin, and C. Lamberti, "Template burning inside TS-1 and Fe-MFI molecular sieves: an in situ XRPD study," Journal of the American Chemical Society, vol. 125, no. 47, pp. 14549-14558, 2003.

[28] G. Agostini, C. Lamberti, L. Palin et al., "In situ XAS and XRPD parametric Rietveld refinement to understand dealumination of Y zeolite catalyst," Journal of the American Chemical Society, vol. 132, no. 2, pp. 667-678, 2010.

[29] R. Caliandro, D. Chernyshov, H. Emerich et al., "Patterson selectivity by modulation-enhanced diffraction," Journal of Applied Crystallography, vol. 45, no. 3, pp. 458-470, 2012.

[30] M. Milanesio, E. Conterosito, D. Viterbo, L. Perioli, and G. Croce, "New efficient intercalation of bioactive molecules into layered double hydroxide materials by solid-state exchange: an in situ XRPD study," Crystal Growth \& Design, vol. 10, no. 11, pp. 4710-4712, 2010.

[31] E. Conterosito, W. Van Beek, L. Palin et al., "Development of a fast and clean intercalation method for organic molecules into layered double hydroxides," Crystal Growth \& Design, vol. 13, no. 3, pp. 1162-1169, 2013.

[32] G. R. Williams, A. I. Khan, and D. O. Hare, "Mechanistic and kinetic studies of guest ion intercalation into layered double hydroxides using time-resolved, in-situ X-ray powder diffraction," in Layered Double Hydroxides, X. Duan and D. G. Evans, Eds., vol. 119 of Structure and Bonding, pp. 161-192, Springer, Berlin, Heidelberg, 2005.

[33] S. Majoni and J. M. Hossenlopp, “Anion exchange kinetics of nanodimensional layered metal hydroxides: use of isoconversional analysis," The Journal of Physical Chemistry A, vol. 114, no. 49, pp. 12858-12869, 2010.
[34] L. Palin, R. Caliandro, D. Viterbo, and M. Milanesio, "Chemical selectivity in structure determination by the time dependent analysis of in situ XRPD data: a clear view of Xe thermal behavior inside a MFI zeolite," Physical Chemistry Chemical Physics, vol. 17, no. 26, pp. 17480-17493, 2015.

[35] P. Guccione, L. Palin, M. Milanesio, B. D. Belviso, and R. Caliandro, "Improved multivariate analysis for fast and selective monitoring of structural dynamics by: in situ X-ray powder diffraction," Physical Chemistry Chemical Physics, vol. 20, no. 4, pp. 2175-2187, 2018.

[36] L. Palin, E. Conterosito, R. Caliandro et al., "Rational design of the solid-state synthesis of materials based on poly-aromatic molecular complexes," CrystEngComm, vol. 18, no. 31, pp. 5930-5939, 2016.

[37] W. Van Beek, O. W. Safonova, G. Wiker, and H. Emerich, "SNBL, a dedicated beamline for combined in situ X-ray diffraction, X-ray absorption and Raman scattering experiments," Phase Transitions, vol. 84, no. 8, pp. 726-732, 2011.

[38] P. Kraft, A. Bergamaschi, C. Broennimann et al., "Performance of single-photon-counting PILATUS detector modules," Journal of Synchrotron Radiation, vol. 16, no. 3, pp. 368-375, 2009.

[39] W. Van Beek, H. Emerich, A. Urakawa et al., "Untangling diffraction intensity: modulation enhanced diffraction on $\mathrm{ZrO} 2$ powder," Journal of Applied Crystallography, vol. 45, no. 4, pp. 738-747, 2012.

[40] E. Conterosito, L. Palin, R. Caliandro, W. van Beek, D. Chernyshov, and M. Milanesio, " $\mathrm{CO}_{2}$ adsorption in $\mathrm{Y}$ zeolite: a structural and dynamic view by a novel principal-component-analysis-assisted in situ single-crystal X-ray diffraction experiment," Acta Crystallographica Section $A$, vol. 75, no. 2, 2019.

[41] A. A. Coelho, "TOPAS and TOPAS-Academic: an optimization program integrating computer algebra and crystallographic objects written in C++," Journal of Applied Crystallography, vol. 51, no. 1, pp. 210-218, 2018.

[42] J. D. Hancock and J. H. Sharp, "Method of comparing solid-state kinetic data and Its application to the decomposition of kaolinite, brucite, and BaCO3," Journal of the American Ceramic Society, vol. 55, no. 2, pp. 74-77, 1972.

[43] R. Caliandro and B. D. Belviso, "RootProf: software for multivariate analysis of unidimensional profiles," Journal of Applied Crystallography, vol. 47, no. 3, pp. 1087-1096, 2014.

[44] E. Conterosito, L. Palin, D. Antonioli et al., "Structural characterisation of complex layered double hydroxides and TGA-GC-MS study on thermal response and carbonate contamination in nitrate- and organic-exchanged hydrotalcites," Chemistry - A European Journal, vol. 21, no. 42, pp. 14975-14986, 2015. 


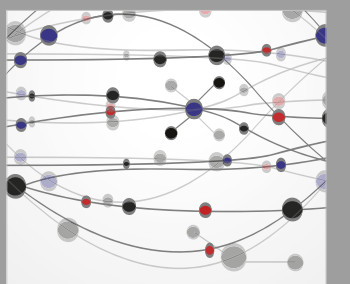

The Scientific World Journal
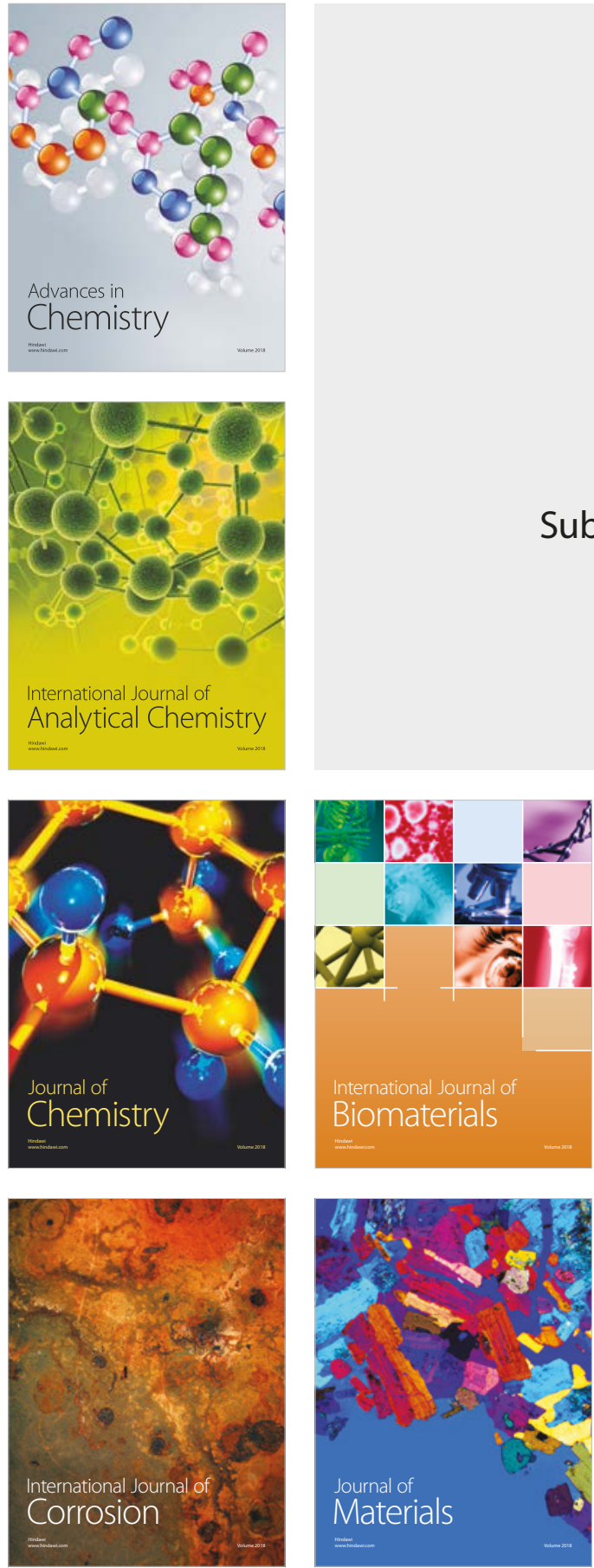

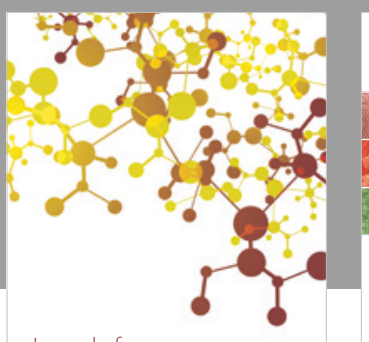

Journal of

Applied Chemistry
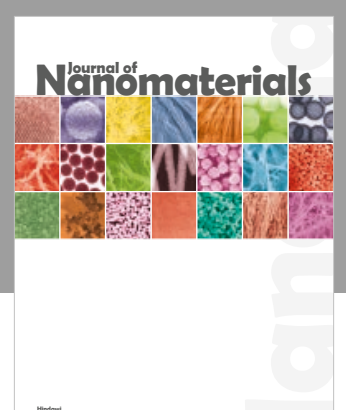

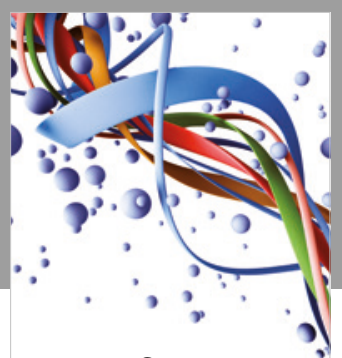

Scientifica

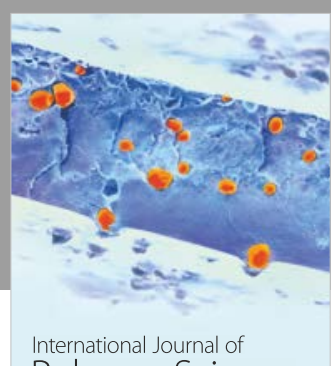

Polymer Science

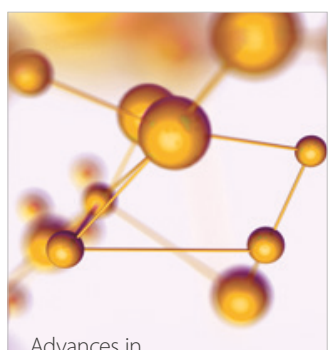

Physical Chemistry
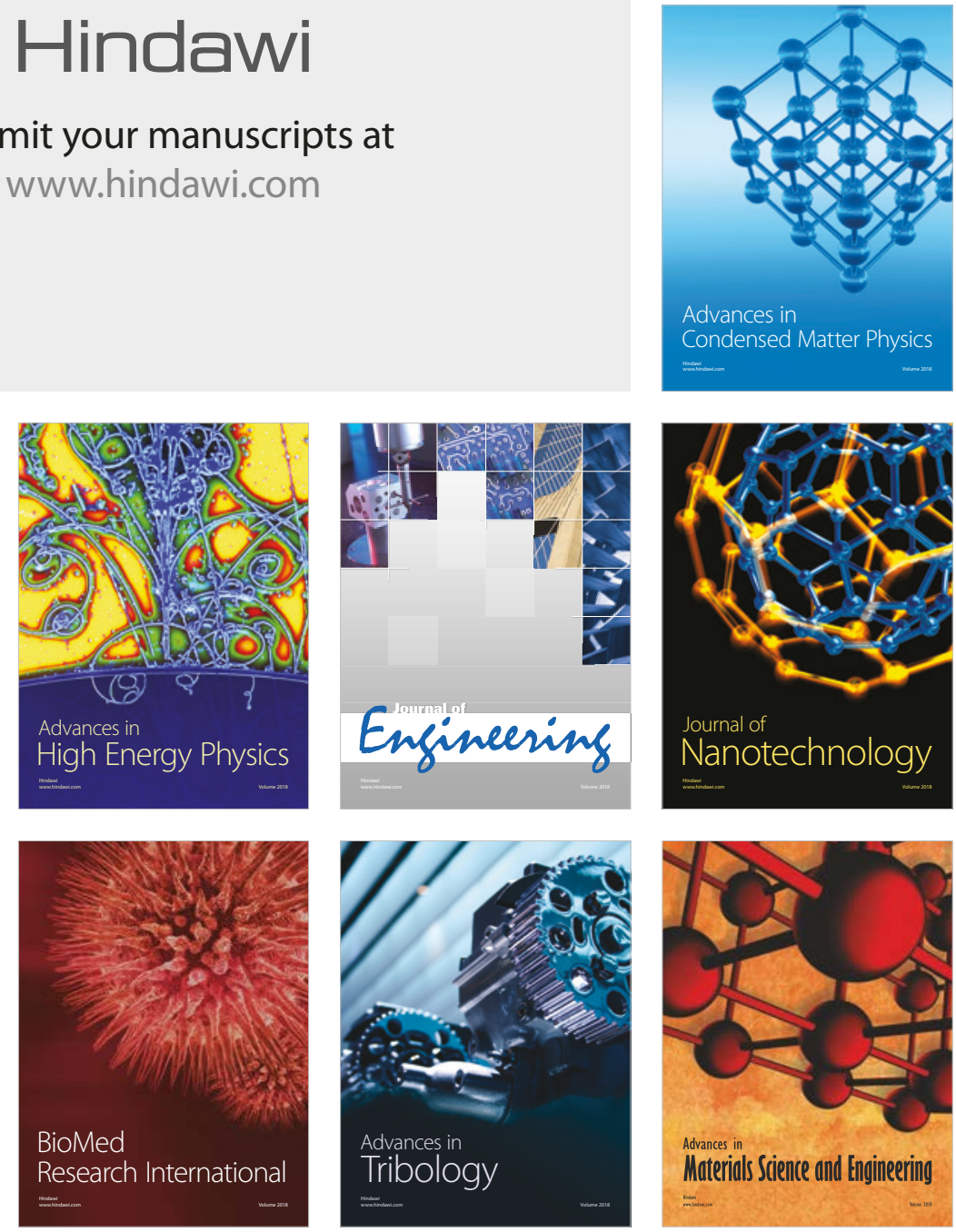